\title{
ACUTE CALCIFIC SYNOVITIS OF THE KNEE
}

\author{
T. S. Mann, Glasgow, Scotland \\ From the Orthopaedic Department, Bridge of Earn Hospital, Perthshire
}

Degenerative osteoarthritis is a cause of joint pain common in the elderly. The knee joint is often affected and minor trauma may produce an exacerbation of symptoms. In a proportion of such patients painful symptoms are accompanied by synovitis and swelling of the joint. A clinical syndrome of acute synovitis of large joints, occurring in elderly persons, has been described by McCarty, Kohn and Faires (1962), who concluded that the synovitis was caused by crystal irritation of the synovia.

This paper reports five patients who, it is thought, suffered acute inflammation of the knee induced by calcium irritation. A simple approach to diagnosis and treatment is suggested.

\section{CASE REPORTS}

Case 1-A woman of sixty-five was admitted to hospital because of pain and swelling of the left knee for two days. There was no history of injury. The knee was tense, tender and warm, suggesting acute septic arthritis. The joint was aspirated and fifty millilitres of yellow opalescent fluid were removed: pain was immediately relieved. The patient remained in bed wearing a

TABLE I

Results OF INVESTIGATIONS IN CASE 1

\begin{tabular}{|c|c|c|c|}
\hline Time interval & $\begin{array}{c}\text { Erythrocyte } \\
\text { sedimentation rate } \\
\text { (millimetres in } \\
\text { the first hour) }\end{array}$ & $\begin{array}{c}\text { Synovial fluid } \\
\text { calcium } \\
\text { (milligrams } \\
\text { per cent) }\end{array}$ & $\begin{array}{c}\text { Serum calcium } \\
\text { (milligrams } \\
\text { per cent) }\end{array}$ \\
\hline On admission . & 29 & $\begin{array}{c}26 \\
\text { (left knee) }\end{array}$ & $10 \cdot 9$ \\
\hline After one week & 33 & $\begin{array}{c}24 \\
\text { (right knee) }\end{array}$ & - \\
\hline After one year. & 15 & $\begin{array}{c}5 \cdot 7 \\
\text { (left knee) }\end{array}$ & $9 \cdot 7$ \\
\hline
\end{tabular}

supporting bandage and practising quadriceps exercises. One week later the right knee became similarly swollen and tense: once more opalescent fluid was removed and symptoms were relieved.

After this the patient remained completely symptom-free for three months, but later mild aches and pains were felt in the knees. There were no other joint symptoms. A year later she was seen with a symptomless effusion of the left knee and a small quantity of clear fluid was removed. Radiological examination revealed light calcification of all four semilunar cartilages and of the articular cartilage of the knees. The triangular fibrocartilage of the wrists also showed calcification. The results of haematological and biochemical examination are shown in Table I. When symptoms were acute the erythrocyte sedimentation rate was raised and the level of calcium in the synovial fluid was found to be greatly increased. Other investigations were normal.

Case 2-A farmer of sixty-seven complained of pain and swelling of the right knee for ten weeks. There was no history of injury. The patient was first seen by his family doctor who noted swelling of the knee: rest and analgesics were prescribed. Symptoms were settling by 
the time he reported to hospital, but the knee was still swollen. Yellow opalescent fluid was aspirated. A year later the patient declared that he had been symptom-free since the time of the aspiration. No other joint had ever given symptoms. Radiological examination showed moderate degenerative changes in both knee joints and there was diffuse calcification in all four menisci and upon the surfaces of the articular cartilage (Fig. 1). Light calcification was present in the fibrocartilages of the acromio-clavicular and radio-carpal joints.

Although this patient was seen when symptoms were lessening, the erythrocyte sedimentation rate was raised to 20 millimetres in the first hour and the synovial fluid calcium content was increased to 11.8 milligrams per cent. The serum calcium was normal at 8.6 milligrams per cent.

Case 3-A man of seventy-three sustained a minor

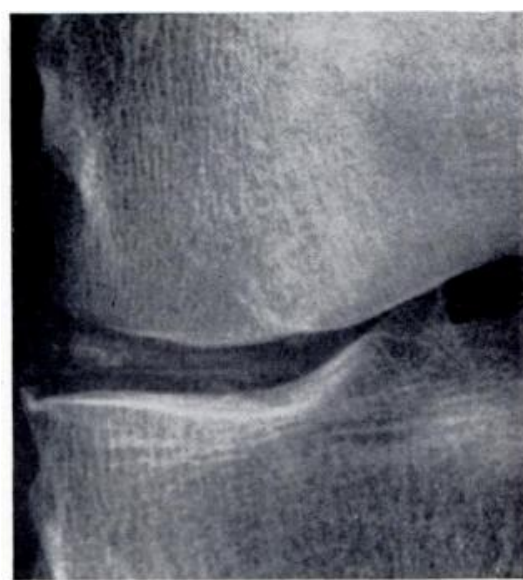

FiG. 1

Case 2-Radiograph of the knee. Calcification is shown in the meniscus and a thin layer of calcium is shown on the surface of the articular cartilage.

twist of his left knee and two days later came to hospital with a tense swollen joint. One hundred millilitres of yellow fluid were removed and the symptoms settled. The effusion recurred and two further aspirations were carried out in the next ten days. A year later he reported that he had had no further knee symptoms and that no other joint had ever given trouble. Radiographs showed calcification of all four semilunar cartilages, more marked in the left knee. There was calcification of the triangular fibrocartilages of the wrist (Fig. 2), the intervertebral discs and the symphysis pubis (Fig. 3), but there was no radiological evidence of calcification of articular cartilage. The calcium content of the synovial fluid was measured only at the third aspiration, when it was found to be 11 milligrams per cent, compared with a serum calcium level of $9 \cdot 1$ milligrams per cent. Further biochemical estimations were normal

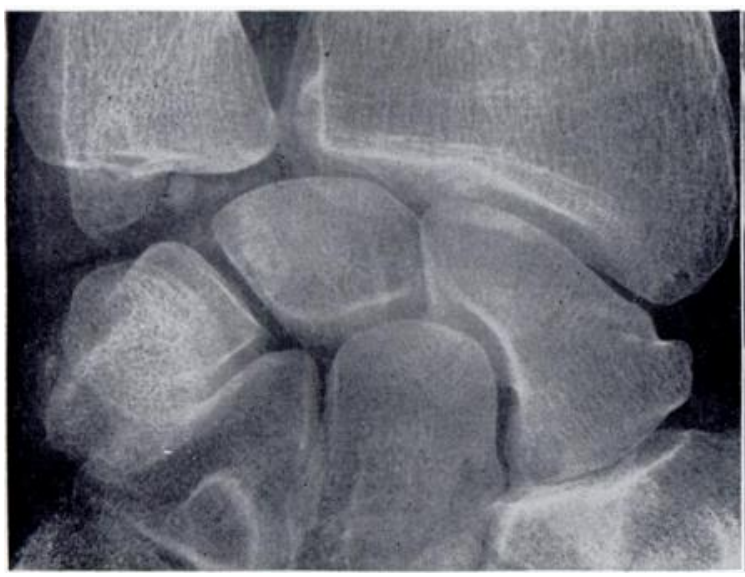

Fig. 2

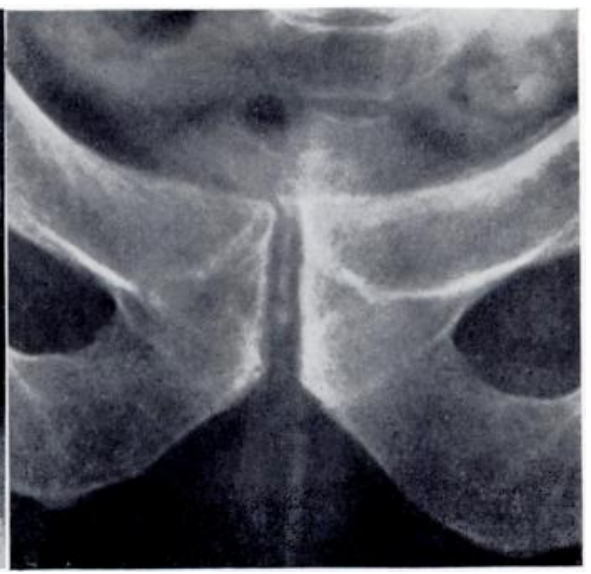

FIG. 3

Case 3. Figure 2-Radiograph of the radiocarpal joint, showing diffuse calcification of the triangular fibrocartilage. Figure 3-Radiograph of the pubis, showing calcification in the symphysis.

Case 4-A man of fifty-five was admitted to hospital with an acute synovitis of the left knee. Sixty-five millilitres of thick straw-coloured fluid were aspirated from the joint and the symptoms settled. Because of a clinical resemblance to acute septic arthritis a course of penicillin was given. A year later he was again admitted with an acute synovitis of the right 
knee, and the symptoms settled after fluid had been withdrawn. Eighteen months later he presented with mild pain under both heels: he had had no further knee symptoms.

During the acute episodes the erythrocyte sedimentation rate was considerably raised (40 millimetres and 45 millimetres in the first hour). The serum uric acid content was found to be normal on several occasions. The synovial fluid was not examined biochemically.

TABLE II

Radiological Evidence of Joint Calcification in the Five Patients

\begin{tabular}{|c|c|c|c|c|c|}
\hline \multirow{2}{*}{ Region } & \multicolumn{5}{|c|}{ Case number } \\
\hline & 1 & 2 & 3 & 4 & 5 \\
\hline \multicolumn{6}{|l|}{ FIBROCARTILAGE } \\
\hline Knee. & + & + & + & + & + \\
\hline Acromio-clavicular joint & - & + & 0 & $n$ & + \\
\hline Radiocarpal joint & + & + & + & + & 0 \\
\hline Intervertebral discs & 0 & 0 & + & - & + \\
\hline Symphysis pubis. & 0 & 0 & $\div$ & 0 & $\therefore$ \\
\hline \multicolumn{6}{|l|}{ ARTICULAR CARTILAGE } \\
\hline Knee. & + & + & - & + & + \\
\hline Sacro-iliac joint & 0 & 0 & 0 & 0 & t \\
\hline
\end{tabular}

+ calcification present; - calcification not present; 0 suitable radiographs not available.

Radiographs of the knees revealed slightly worn joints; there was diffuse calcification of all four menisci and a fleck of calcification could be seen on the articular cartilage of one femoral condyle. There was some calcification in one supraspinatus tendon and in the triangular fibrocartilage of one wrist. Bilateral calcaneal spurs were present.

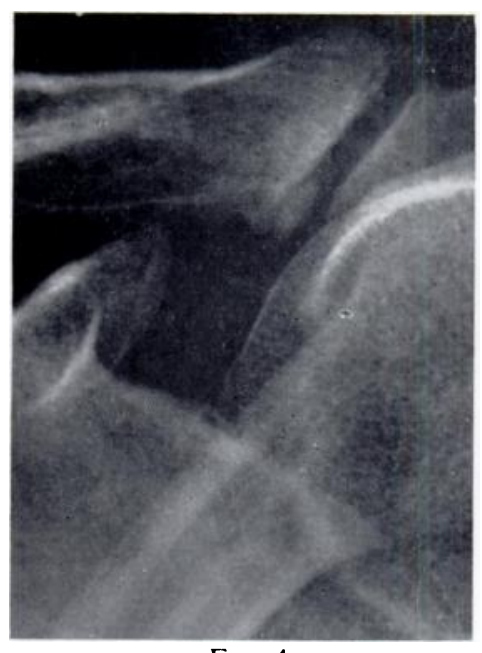

FIG. 4

Case 5-A man of seventy-two complained of acute pain in his left hip. He then developed pain in his left knee with a tense, warm effusion. The erythrocyte sedimentation rate was 60 millimetres in the first hour. With bed rest the symptoms settled and there was then no indication to aspirate the knee. Radiographs revealed calcification of the menisci in both knees and it seems certain that symptoms were due to a calcium-induced synovitis. There was radiological evidence of calcification in the symphysis pubis, the intervertebral discs, the fibrocartilage of the acromio-clavicular joints (Fig. 4) and the articular cartilage of the sacro-iliac joints.

\section{Fig. 4}

Case 5-Radiograph of the acromio-clavicular joint showing calcification within the intra-articular fibrocartilage.

\section{DISCUSSION}

Calcification of the menisci of the knee is a not unusual radiological finding. In such a case symptoms are usually attributed to degenerative arthritis. The concept of a calciuminduced synovitis has been slow to develop. Weaver (1942) discussed calcification of the menisci, reviewing seventy-six patients reported in the literature and describing two others 
in detail. He described two kinds of calcification: a primary type occurring in older patients which usually involves both menisci of both knees, and a secondary type which follows injury to a single meniscus in younger patients. He reported that patients with primary calcification usually showed some evidence of hypertrophic arthritis, and he described calcification of the intervertebral discs as an associated radiological finding. Effusion into the knee was not especially discussed.

Calcification of hyaline articular cartilage is not common. It was described by Israelski (1931) who published radiographs of the knee of a patient of sixty. The menisci were diffusely calcified and the articular surfaces were outlined by a narrow radio-opaque band. Symptoms were of joint stiffness without swelling.

Codman (1934) described how acute subacromial bursitis occurs in association with calcified deposits in the supraspinatus tendon. He stated that calcification of the tendon is secondary to a degenerative process and he discussed how rupture of calcareous material into the bursa produces acute bursitis. He emphasised the severity of the symptoms in such cases and pointed out the clinical resemblance to an acute septic bursitis.

Calcification in the capsule of the hip in association with acute synovitis was reported by Jones (1955). He suggested that the calcium deposit ruptured into the joint, provoking an irritant reaction. On the other hand, Bunjé and Cole (1956) described a woman of thirty-one in whom intermittent fever, joint swelling and pain were associated with calcification in the fibrocartilage and hyaline cartilage of many joints and concluded that the calcium deposition was the result of a recurrent mild arthritis.

The term "chondrocalcinosis articularis" has been used to describe the association of multiple and recurrent joint pains with radiological evidence of calcification in fibrocartilage and hyaline cartilage. Žitñan and Šitaj (1963) studied twenty-seven patients in detail. In twenty-one evidence of a familial disorder was found and a sex-linked chromosome inheritance was suggested. It was found that calcification preceded the clinical manifestations of arthritis. Arthralgia and arthritis commonly began in the third or fourth decade of life. There was no evidence of metabolic disorder and the authors postulated a hereditary disturbance of articular intercellular ground substance.

A similar clinical and radiological picture was described in older patients by McCarty, Kohn and Faires (1962). Because of a clinical resemblance to synovial gout, they called the syndrome " pseudogout." The knee joint was most commonly involved; synovial effusion was a feature and symptoms were often recurrent. No family predisposition was noted. Calcium pyrophosphate crystals were identified in the synovial fluid of all patients and it was concluded that the syndrome was an example of crystal-induced synovitis. It was thought that pain occurred only when a critical level of calcium crystals had been deposited.

Table III presents a classification of the causes of calcification within a joint. There are two main groups, metastatic and dystrophic. In metastatic calcification calcium is laid down because of a generalised metabolic disorder, usually accompanied by raised calcium levels in the serum. Keynes and Taylor (1933) described the joint changes that occur in hyperparathyroidism. The most significant feature is calcification of the synovial membrane and joint capsule, and in the knee the quadriceps tendon is commonly involved. In a discussion of the joint lesions in hyperparathyroidism Bywaters, Dixon and Scott (1963) described two elderly patients whose knees showed radiological evidence of calcification in the menisci and the articular cartilage. In both patients synovial calcification was also present.

In dystrophic calcification the levels of calcium and phosphate in the blood are normal, but deposition of calcium is promoted by a general or local tissue disorder. Watson Jones and Roberts (1934) pointed out that pathological calcification is seen in tissues where metabolism is low. They considered that calcification of the semilunar cartilages was evidence of a degenerative change in relatively avascular fibrocartilaginous tissue. Selye, Goldie and Strebel (1963) stated that the calcium avidity of soft tissues increases with advancing age.

VOl. $48 \mathrm{~B}$, NO. 1, FEBRUARY 1966 
TABLE III

Causes of Deposition of Calcium in Joints

\section{Dystrophic}

1) Degeneration: primary . Increased avidity for calcium of ageing tissues secondary . A late effect of trauma

2) Chondrocalcinosis articularis Calcium deposition conditioned by a familial disturbance of tissue metabolism

3) Ochronosis . . . Accelerated degeneration of tissues caused by the presence of abnormal amino acids (Thompson and King 1964)

4) Calcinosis universalis . . Local or general inflammation or degeneration resulting in deposition of calcium

\section{Metastatic}

Disorders, such as hyperparathyroidism, where calcium deposition is secondary to a raised blood content of calcium

TABLE IV

InCrease of the Calcium Content of Synovial Fluid in Acute Effusions of the KneE (Five Patients with Dystrophic Calcification)

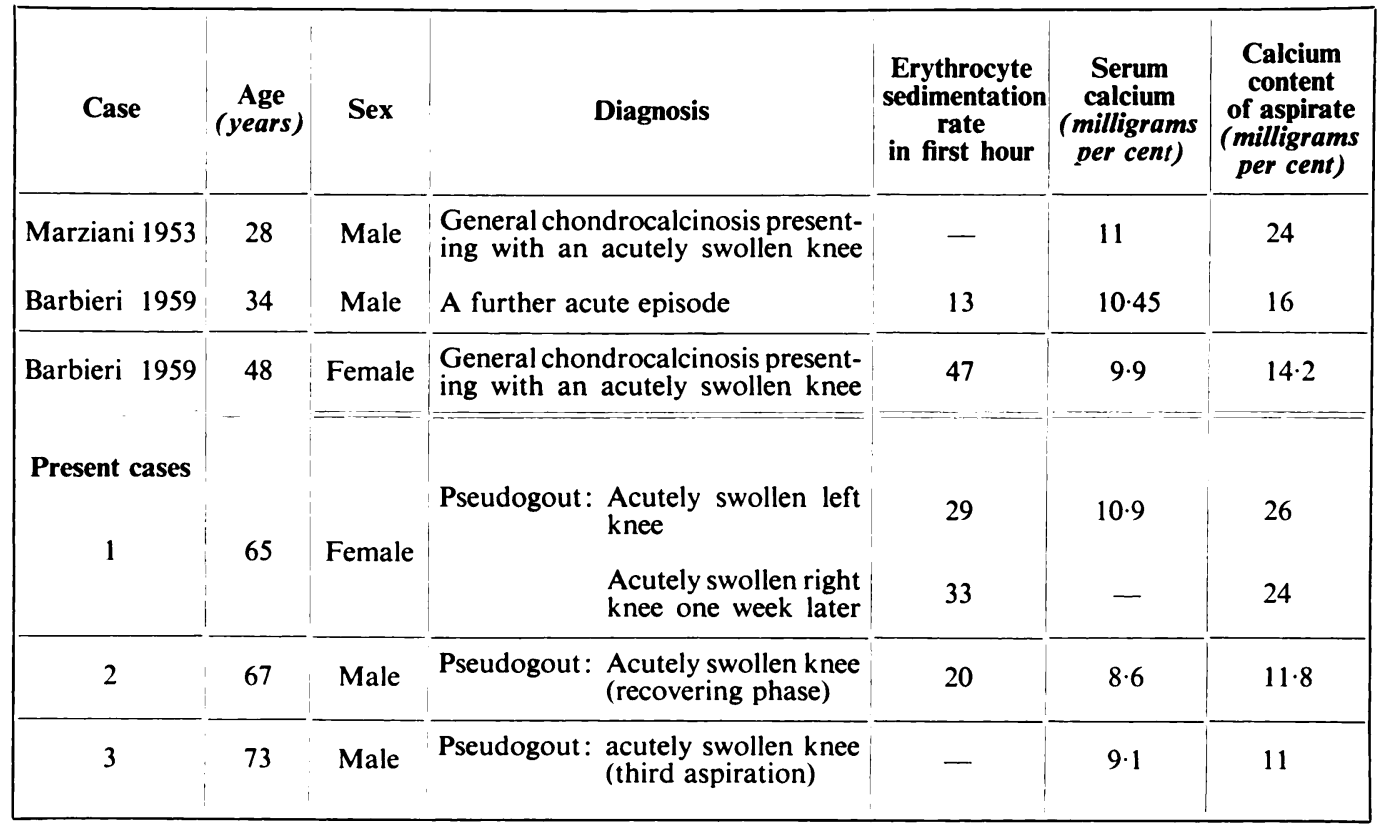

In normal joint fluid the calcium content is less than that in the serum. Ropes and Bauer(1953) found the relationship for normal joints to be $\sqrt{\text { serum calcium }}=0.87$. In patients with degenerative joint disease they found the ratio to be 0.92 . Significantly, in a study of 1,500 joint fluids, in only five were the calcium levels higher in the synovial fluid than in the serum: three patients suffered from hypervitaminosis $D$ and two had unspecified connective tissue disease. In patients with osteoarthritis Kling (1938) reported the occasional finding of high calcium values in synovial fluid (up to 14 milligrams per cent), in the presence of normal serum values. Similar values were found in patients suffering from quaternary syphilis. 
Biochemical studies in patients with dystrophic calcification show normal blood levels of calcium and phosphate. There have been few recordings of synovial fluid content. Marziani (1953) described a young man with an acutely swollen knee, calcification of the menisci, and an elevated synovial fluid calcium content ( 24 milligrams per cent). Six years later Barbieri (1959) saw this patient with a further effusion: the general radiological features of chondrocalcinosis articularis were noted, and the synovial fluid calcium content was raised (17 milligrams per cent). Barbieri also described a middle-aged woman with similar clinical, radiological and biochemical features. Table IV outlines the features of these two patients for comparison with three of the five reported in this paper. Five patients are thus summarised, all of whom experienced acute swelling of one or both knee joints; in all of them there was evidence of local or general dystrophic joint calcification and the content of calcium in acute synovial effusion was found to be higher than the content in the serum.

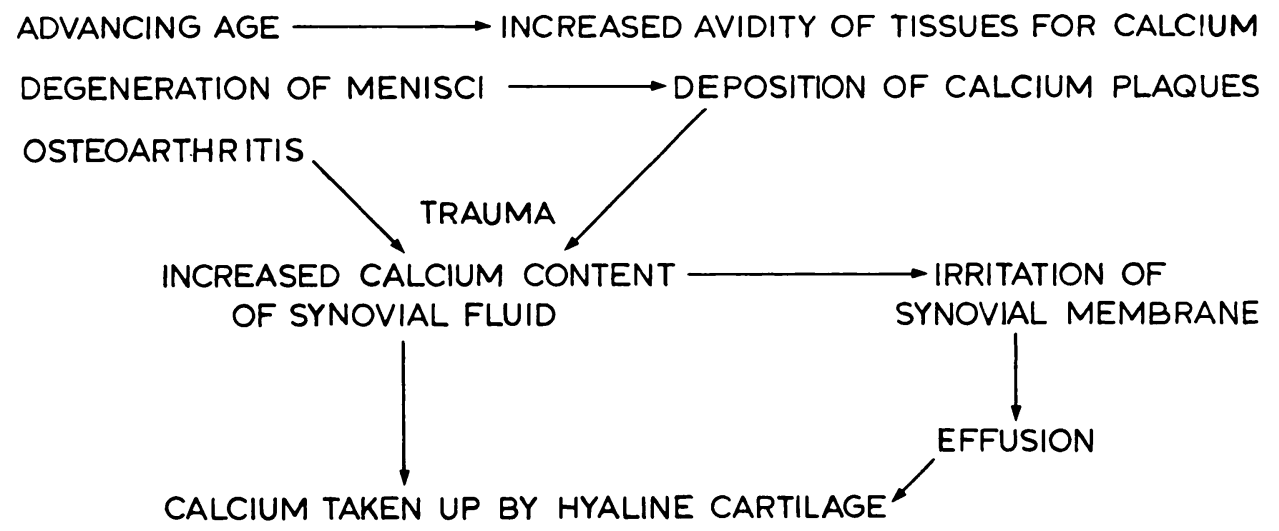

FIG. 5

Showing the sequence of calcification in the older joints.

In the absence of metabolic disease the most likely causes of joint calcification are a primary degeneration of the joint or a familial disturbance of the tissue. The relationship between these two groups is not entirely clear, and the spectrum of calcium-induced synovitis appears to spread from the young patient with familial polyarthrosis to the older with an ageing knee. The five patients presented in this paper all had a degree of degenerative arthritis of the affected joint: they presented with the clinical features of pseudogout rather than with the radiological syndrome of chondrocalcinosis articularis.

It seems that the sequence of events in degenerative joints is as follows: 1) Against a background of wear and tear the ageing avascular meniscus takes up calcium from the synovial fluid and calcium plaques are formed. 2) Slight trauma to the joint then causes release of calcium from the menisci. 3) Or calcium crystals may be disturbed from eburnated bone or, more probably, from hypertrophic ossification at the edge of the articular surfaces. If the joint has been the site of old trauma, new bone may have formed in other sites related to the synovial spaces. 4) The presence of a high level of calcium in the joint then causes irritation of the synovial membrane, producing an effusion. 5) After this the synovitis settles. Calcium is deposited on the surfaces within the joint. The articular cartilage acts to promote an ion exchange (Thompson and King 1964) and calcification of the superficial layers takes place (Fig. 5).

If this sequence of events is correct, radiological evidence of calcification of cartilage is not needed for diagnosis of calcium-induced synovitis. Any worn joint may suffer this kind of irritation.

VOL. 48 B, NO. 1, FEBRUARY 1966 
Diagnosis-The diagnosis of chondrocalcinosis articularis was made by Žitñan (1963) on radiological findings, even if the patient was symptom-free. McCarty and his colleagues proved pseudogout by the use of phase contrast and polarised light microscopy to identify calcium pyrophosphate crystals in the synovial effusion, and they preferred also to use $\mathrm{x}$-ray diffraction crystallography.

Since acute symptoms are due to calcium irritation it is reasonable to base diagnosis upon synovial calcium levels. Metastatic calcification can be excluded by radiographs and normal serum levels of calcium, phosphate and alkaline phosphatase. Ochronosis can be excluded by examination of the urine for homogentisic acid. The serum uric acid should be estimated. The joint should be aspirated and the fluid calcium level measured. In the presence of acute symptoms this level always seems to be raised. At a later stage it would be reasonable to regard the result as significant if the calcium content of the synovial fluid is found to be greater than that of the serum.

Treatment-In four of the five patients reported in this paper a diagnostic aspiration was used to drain all the fluid from the affected joint. Six joints were thus treated and in all immediate relief was felt and the acute symptoms settled. A compression bandage was applied after aspiration and quadriceps exercises started. Three patients spent a short period in hospital after aspiration: one (Case 2) was treated as an out-patient. After a year only one joint had given recurrent symptoms and in that one a small chronic effusion was present.

Apart from its place in diagnosis, aspiration is a logical therapeutic measure in any case of joint effusion: when fluid has been removed muscle control is more easily regained and mobility can be recovered. In calcium-induced synovitis the acute symptoms arise from the irritative effect of the calcium crystals: the synovitis itself is a body defence mechanism which dilutes and counteracts the irritant. Removal of as much fluid as possible takes away most of the free calcium, stops the irritation and may indeed prevent the deposition of further calcium upon the articular surfaces. The compression bandage acts as a splint and prevents further stirring up of calcium within the joint. When symptoms have settled, the practice of quadriceps exercises helps regain control of the knee. Major trauma is not an etiological factor in these cases, but many if not all patients admit to a minor twist or strain: good musculature more easily controls the joint and helps to guard against minor injury.

No specific treatment has been devised to prevent recurrence or to control polyarthralgia, but it is logical to prescribe analgesics where joint pain persists in the absence of synovitis. It is not at present possible to reverse dystrophic calcification or remove the calcium deposits.

\section{CONCLUSIONS}

Joint inflammation caused by calcium irritation is more common than is generally realised. The diagnosis should be remembered whenever an adult patient is seen with acute joint symptoms. The early features are acute irritation and in an accessible joint synovial effusion will be detected. The onset is often very acute and may resemble a septic arthritis: pain is severe and immobilises the joint and there is local heat and tenderness. If the patient is seen later a subacute synovitis may be present. A history of previous episodes may be given and several joints may be involved. There is a clinical resemblance to synovial gout and rheumatoid arthritis. The erythrocyte sedimentation rate is raised in the acute stage.

Radiological examination usually reveals calcification within the joint. Radiological features are frequently bilateral; many joints may be involved; and changes may be found in joints in which symptoms have not occurred. Fibrocartilage is more frequently involved than hyaline cartilage, and the knees and radiocarpal joints are the most likely to show radiological change (Table II). The fibrocartilage of the knee, radiocarpal and acromio-clavicular joints, the symphysis pubis and the intervertebral discs show patchy or diffuse calcification (Figs. 1 to 4). The hyaline cartilage of joints shows a fine surface calcification. In older patients 
degenerative changes are often seen, or there may be dystrophic calcification at the site of previous trauma to capsule or bone.

\section{SUMMARY}

1. Five elderly patients who suffered acute synovitis of one or both knee joints are reported.

2. All showed radiological evidence in several joints of cartilage calcification.

3. It is suggested that the synovitis in each case was due to calcium irritation of the synovial membrane.

4. In three of the patients it is shown that the synovial fluid calcium content was raised during the acute attack.

5. In all patients acute symptoms were relieved by aspiration of the effusion.

I am indebted to Mr Ian Smillie and Mr James Hutchison for their helpful criticism during the writing of this paper. I am grateful to Mr Adam Tilp-Zielinski, A.R.P.S., for reproduction of the Figures.

\section{REFERENCES}

BARBIERI, M. (1959): Incrostazione calcarea sistemica delle cartilagini articolari. Clinica Ortopedica, 11, 360. Bunjé, H., and Cole, W. R. (1956): Calcification of Articular Cartilage. Journal of Bone and Joint Surgery, 38-B, 874.

Bywaters, E. G. L., Dixon, A. St J., and Scott, J. T. (1963): Joint Lesions of Hyperparathyroidism. Annals of the Rheumatic Diseases, 22, 171.

Codman, E. A. (1934): The Shoulder, pp. 68-70. Boston: Todd.

ISRAELSK, M. (1931): Meniscus Calcification. American Journal of Roentgenology, 25, 85.

JONES, G. B. (1955): Acute Episodes with Calcification around the Hip Joint. Journal of Bone and Joint Surgery, 37-B, 448.

Keynes, G., and Taylor, H. (1933): A Case of Parathyroid Tumour. British Journal of Surgery, 21, 20.

KuING, D. H. (1938): The Synovial Membrane and the Synovial Fluid. Los Angeles: Medical Press.

McCarty, D. J., Jun., KohN, N. N., and FaIREs, J. S. (1962): The Significance of Calcium Phosphate Crystals in the Synovial Fluid of Arthritic Patients: The "Pseudogout Syndrome." Annals of Internal Medicine, 56, 711.

Marziani, R. (1953): L'Incrostazione calcarea sistemica delle cartilagini articolari. Archivio Putti di Chirurgia degli Organi di Movimento, 3, 220.

Ropes, M. W., and BAUER, W. (1953): Synovial Fluid Changes in Joint Disease. Cambridge, Mass.: Harvard University Press.

Selye, H., Goldie, I., and Strebel, R. (1963): Calciphylaxis in Relation to Calcification in Periarticular Tissues. Clinical Orthopaedics and Related Research, 28, 181.

Thompson, R. H. S., and KING, E. J. (1964): Biochemical Disorders in Human Disease. Second edition. London: J. \& A. Churchill Ltd.

Watson Jones, R., and RoBerts, R. E. (1934): Calcification, Decalcification, and Ossification. British Journal of Surgery, 21, 461.

WeAVER, J. B. (1942): Calcification and Ossification of the Menisci. Journal of Bone and Joint Surgery, $24,873$.

Żrț́an, D., and Sitaj, S. (1963): Chondrocalcinosis Articularis. Annals of the Rheumatic Diseases, $22,142$. 